\title{
Thickness and Connectivity in Graphs
}

\author{
Arthur M. Hobbs and J. W. Grossman \\ Institute for Applied Technology, National Bureau of Standards, \\ Washington, D.C. 20234
}

(June 25, 1968)

\begin{abstract}
A graph $G$ has thickness $t$ if and only if $t$ is the smallest number of planar subgraphs of $G$ whose union is $G$. A thickness-minimal, or t-minimal, graph is a graph of thickness $t$ with no proper subgraph of thickness $t$. In this paper we show that, for every $t$, a cut set in a $t$-minimal graph can have no fewer than $t$ edges. This theorem suggests that the connectivity of a $t$-minimal graph is bounded from below by $t$. We disprove this suggestion for every $t>2$ by constructing a graph which contains a 2-connected $t$-minimal graph. Two theorems extend this result and develop additional properties of the construction method. In addition, we show that the connectivity of a graph of thickness $t$ is at most $6 t-1$ and that the thickness of $K_{22}$ is 4 . Finally, we make four conjectures about graphs of thickness $t$.
\end{abstract}

Key Words: Conjectures, connectivity, cut-set, graph theory, mathematics, minimality, thickness of graphs.

\section{Definitions and Previous Results}

A graph is a finite set of points, called vertices, together with a finite set of line segments, called edges, such that each end point of an edge is a vertex, no edge meets more than two vertices, and edges meet only at end points. If two edges share an end point, they are adjacent. The end points of an edge are said to be joined by the edge. A subgraph of a graph $G$ is a subset of the edges and vertices of $G$ which is again a graph.

A graph is complete if and only if every pair of vertices is-joined by an edge; a complete graph with $n$ vertices is denoted by $K_{n}$. A graph $G$ is bipartite if and only if there is a separation of the vertices of $G$ into two disjoint sets $A$ and $B$ such that every edge of $G$ joins a vertex in $A$ with a vertex in $B$. A bipartite graph is complete if and only if every vertex in $A$ is joined to every vertex in $B$ by an edge; such a graph is denoted by $K_{m, n}$, where $m$ is the number of vertices in the set $A$ and $n$ is the number of vertices in the set $B$.

A graph is planar if and only if it can be drawn in the plane in such a way that no two distinct points of the graph occupy the same point of the plane. The union of subgraphs $H_{1}, H_{2}, \ldots ., H_{n}$ of a graph $G$ is the subgraph of $G$ consisting of all edges and all vertices from the $H_{i}$. The thickness $\theta(G)$ of a graph $G$ is defined [5] as the minimum number of planar subgraphs whose union is $G$. A thickness-minimal, or t-minimal, graph is a graph of thickness $t$ which contains no proper subgraph of thickness $t$.

A (simple) path in $G$ is a vertex, a single edge, two adjacent edges, or a subset $e_{1}, e_{2}, \ldots ., e_{n}$, $n>2$, of the edges of $G$ such that $e_{i+1}$ joins an end point of $e_{i}$ with an end point of $e_{i+2}, i=1,2, \ldots$, $n-2$, and no vertex is the end point of more than two edges of the path. The path is said to join any two vertices met by edges of the path. A circuit is a path such that $e_{1}$ and $e_{n}$ are adjacent. A graph or subgraph $G$ is connected if and only if for any two vertices of $G$ there exists a path in $G$ joining the two vertices. The girth of a graph $G$ is the smallest integer $n$ such that there exists a circuit in $G$ having $n$ edges. 
A component of a graph $G$ is a maximal connected subgraph of $G$. The connectivity of a connected graph $G$ is the smallest number of vertices of $G$ whose removal (together with all edges meeting at least one of the vertices) leaves a subgraph of $G$ which has no edges or which is not connected. If $G$ is not connected, it has connectivity 0 . An articulation point of a graph $G$ is a vertex whose removal from $G$ (together with all incident edges) increases the number of components in the remaining graph. A cut set of a graph $G$ is a subset $S$ of the edges of $G$ whose removal disconnects $G$ and such that no proper subset of $S$ has the same property.

W. T. Tutte $[5]^{1}$ proved several theorems which we have found very useful. We state those theorems here with their original numbering:

(3.1) Let $G$ be a graph of thickness $t$. Let $G^{\prime}$ be formed from $G$ by deleting either a single edge or a single vertex with all its incident edges. Then the thickness of $G^{\prime}$ is either $t$ or $t-1$. (In particular, if $G$ is $t$-minimal, then $\theta\left(G^{\prime}\right)=t-1$.)

(3.2) Let $k$ be a nonnegative integer and let $G$ be a graph of thickness $t \geqslant k$. Then there exists a $k$-minimal subgraph of $G$.

(3.3) Every $t$-minimal graph is connected and nonseparable. (A graph is nonseparable if and only if it is connected and contains no articulation points.)

(5.3) For each integer $t \geqslant 2$ there exist infinitely many nonisomorphic $t$-minimal graphs of upper valency $2 t-1$, and of girth greater than any specified integer $N$.

\section{Connectivity and Thickness}

The valency of a vertex of a graph $G$ is the number of edges incident on the vertex. Let $v$ be the least integer occurring as the valency of a vertex of $G$. W. T. Tutte has shown [5] that if $G$ is a $t$-minimal graph, $t>0$, then $v \geqslant t$. The following generalizes Tutte's result:

THEOREM 1: Let $\mathrm{G}$ be $\mathrm{t}$-minimal, $\mathrm{t}>0$, and let $\mathrm{n}$ be the number of edges in a cut set of $\mathrm{G}$. Then $n \geqslant t$.

Proof: Suppose $G$ has a cut set $S$ with $k<t$ edges in it. Let the vertices of $G$ be partitioned into the sets $A$ and $B$ by $S$. Obtain $G^{\prime}$ by removing one edge $e=(a, b) \epsilon S$ from $G$. Since $G$ is $t$-minimal, $\theta\left(G^{\prime}\right)=t-1$, and we can find $t-1$ planar subgraphs $H_{1}, H_{2}, \ldots, H_{t-1}$ of $G^{\prime}$ whose union is $G^{\prime}$. We may assume that no edge of $G^{\prime}$ appears in more than one of the $H_{i}$. There are only $k-1<t-1$ edges of $S$ in $G^{\prime}$, so one of the $H_{i}$, say $H_{1}$, must not contain any edge of $S$. Because $S$ is a cut set of $G, H_{1}$ (which we may assume contains all the vertices of $G$ ) must be disconnected with vertex $a$ in one component and vertex $b$ in some other component. Using stereographic projection, we may draw the component containing vertex $a$ in the plane so that $a$ is on the boundary of the infinite region of the component, and similarly with the component containing vertex $b$. Then a new planar subgraph $H_{1}^{\prime}$ can be formed from $H_{1}$ by joining vertices $a$ and $b$ by edge $e$. But the union of $H_{1}^{\prime}$ together with $H_{2}, \ldots, H_{t-1}$ is $G$, thus giving a partition of $G$ into $t-1$ planar subgraphs, contrary to hypothesis.

Q.E.D.

Given the result of Theorem 1, we might conjecture that the connectivity of a $t$-minimal graph is bounded from below by $t$. However, for all $t>2$, the following propositions show this conjecture to be false.

Let us define an operation on two disjoint graphs $G_{1}$ and $G_{2}$ as follows: choose an edge $e_{i}=\left(a_{i}\right.$, $b_{i}$ ) from graph $G_{i}$, delete edge $e_{i}$ from $G_{i}$, and identify $a_{1}$ with $a_{2}$ and $b_{1}$ with $b_{2}$, obtaining a graph $J\left(G_{1}, G_{2}\right)$ called the join of $G_{1}$ and $G_{2}$. Note that if $G_{1}$ and $G_{2}$ each have connectivity at least 2 , then $J\left(G_{1}, G_{2}\right)$ has connectivity exactly 2 . In general there are many different joins of two graphs, depending on the choice of edges $e_{1}$ and $e_{2}$. However, in this paper we are concerned only with those properties of the join which are independent of the choice of the two edges. Let us denote the vertex resulting from identifying $a_{1}$ and $a_{2}$ by $a$, and that from $b_{1}$ and $b_{2}$ by $b$. The subgraph of $J\left(G_{1}, G_{2}\right)$ corresponding to $G_{1}-\left\{e_{1}\right\}$ we denote by $G_{1}^{\prime}$ and that corresponding to $G_{2}-\left\{e_{2}\right\}$ by $G_{2}^{\prime}$. Since the thickness of a graph is reduced at most 1 by the removal of an edge, it is clear that $\theta\left(J\left(G_{1}, G_{2}\right)\right) \geqslant \max \left(\theta\left(G_{1}\right), \theta\left(G_{2}\right)\right)-1$.

${ }^{1}$ Figures in brackets indicate the literature references at the end of this paper. 
LEMMA: If $\theta\left(\mathrm{G}_{1}\right)=\theta\left(\mathrm{G}_{2}\right)=\mathrm{t}$, then any join of $\mathrm{G}_{1}$ and $\mathrm{G}_{2}$ has thickness $\mathrm{t}$ or $\mathrm{t}+1$.

Proof: Remove vertices $a$ and $b$ and all incident edges from $J\left(G_{1}, G_{2}\right)$. In the resulting graph every component is a proper subgraph of $G_{1}^{\prime}$ or $G_{2}^{\prime}$ and can therefore be partitioned into $t$ or fewer planar subgraphs. Since $K_{2, m}$ is planar for every integer $m$, we can place $a$ and $b$ with their incident edges in one additional plane. Thus, $\theta\left(J\left(G_{1}, G_{2}\right)\right) \leqslant t+1$.

But suppose $J\left(G_{1}, G_{2}\right)$ can be partitioned into $t-1$ planar subgraphs $H_{1}, \ldots, H_{t-1}$. The subgraph $G_{i}^{\prime}$ of $J\left(G_{1}, G_{2}\right)$ together with the additional edge $(a, b)$ is isomorphic to $G_{i}$ for each $i$. Since $G_{i}$ has thickness $t, H_{1} \cap G_{1}^{\prime}$ together with edge $(a, b)$ cannot be planar. Hence in any embedding of $H_{1} \cap G_{1}^{\prime}$ in the plane there is a circuit $C$ composed entirely of edges and vertices of $H_{1} \cap G_{1}^{\prime}$ such that $a$ and $b$ are in different components of the plane if $C$ is removed from it.

Supposing that $a$ and $b$ are not joined by a path in $H_{1} \cap G_{2}^{\prime}$ they must be in separate components thereof. We can then draw each in the plane in such a way as to place $a$ and $b$ on the boundaries of their components and join them by an edge. Thus we obtain a partition of $G_{2}$ into $t-1$ planar subgraphs. Therefore, a path $P$ having more than one edge joins $a$ with $b$ and lies entirely within $H_{1} \cap G_{2}^{\prime}$. Now $P$ must cross $C$ in $H_{1}$. By the Jordan curve theorem, $P$ and $C$ must share a point, and by the definition of planar embedding of graphs, that point must be a vertex of $G$ other than $a$ and $b$. But $a$ and $b$ are the only vertices shared by $G_{1}^{\prime}$ and $G_{2}^{\prime}$. Hence, $\theta\left(J\left(G_{1}, G_{2}\right)\right) \geqslant t$. Q.E.D.

THEOREM 2: For every $\mathrm{t} \geqslant 2$, there exists a $\mathrm{t}$-minimal graph with connectivity 2.

Proof: If $G_{i}$ is $t$-minimal, $G_{i}^{\prime}$ cannot include a $t$-minimal subgraph. But since $\theta\left(J\left(G_{1}, G_{2}\right)\right) \geqslant t$, there exists a $t$-minimal subgraph $K$ contained in $J\left(G_{1}, G_{2}\right)$ (Theorem 3.2 of [5]). Clearly this $t$ minimal subgraph includes one or both of $a$ and $b$, as well as other vertices from each of the $G_{i}^{\prime}$. But Theorem 3.3 of [5] states that $K$ cannot have an articulation point. Therefore, both $a$ and $b$ are contained in $K$, and $K$ has connectivity 2 .

Q.E.D.

The results of Tutte's paper [5] allow us to extend the above theorem much farther. Denote the girth of $G_{i}$ by $g\left(G_{i}\right)$.

THEOREM 3: For each integer $\mathrm{t} \geqslant 2$ and $\mathrm{N} \geqslant 2$, there exists a $\mathrm{t}$-minimal graph of connectivity 2 with girth greater than $\mathrm{N}$.

Proof: $J\left(G_{1}, G_{2}\right)$ cannot have girth less than $\min \left(g\left(G_{1}\right), g\left(G_{2}\right)\right)$, since the only circuits of $G_{i}$ affected by the join are those including edge $\left(a_{i}, b_{i}\right)$, and those circuits are increased by at least one edge in the join. Thus, for all graphs $G_{1}$ and $G_{2}, g\left(J\left(G_{1}, G_{2}\right)\right) \geqslant \min \left(g\left(G_{1}\right), g\left(G_{2}\right)\right)$. By Theorem 5.3 of [5], given an integer $N$, there exists a $t$-minimal graph $G$ with girth greater than $N$. Then $g(J(G, G)) \geqslant g(G)>N$. Since any subgraph of a graph of girth $m$ has girth at least $m$, for any $t$-minimal subgraph $K$ contained in $J(G, G), g(K)>N$, and, by Theorem 2, the connectivity of $K$ is 2 .

Q.E.D.

COROLLARY: For every $\mathrm{t} \geqslant 2$ there exists an infinite class of nonisomorphic t-minimal graphs, each having connectivity 2.

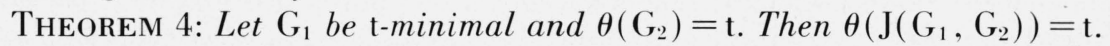

Proof: Partition $G_{2}$ into $t$ planar subgraphs $H_{i}$ such that $a_{2}$ and $b_{2}$ are on the boundary of the same region in one of the subgraphs, say $H_{1}$ (this is possible since $G_{2}$ has thickness $t$ with edge $\left(a_{2}, b_{2}\right)$ present). Remove vertices $a_{1}$ and $b_{1}$ and all incident edges from $G_{1}$. Since $G_{1}$ is $t$-minimal, the resulting graph $G_{1}^{\prime \prime}$ has thickness $t-1$ or less. Partition $G_{1}^{\prime \prime}$ into $t-1$ planar subgraphs and assign one of these subgraphs to each of the $t-1$ planar subgraphs of $G_{22}$ other than $H_{1}$. Now it is trivial to embed $K_{2, n}$ in the plane so that the two vertices in the first subset are on the boundary of the infinite region. Hence we may identify $a_{1}$ with $a_{2}$ and $b_{1}$ with $b_{2}$ in subgraph $H_{1}$ of $G_{2}$, placing all edges joined to either $a_{1}$ or $b_{1}$ in that subgraph. The result is an embedding of $J\left(G_{1}, G_{2}\right)$ (together with edge $(a, b) !)$ in $t$ planes, and by Theorem $2, \theta\left(J\left(G_{1}, G_{2}\right)\right)=t$.

Q.E.D.

Having answered the question of the lower bound for the connectivity of a $t$-minimal graph, it is natural for us to ask for an upper bound for the connectivity of a graph of thickness $t$. Such an upper bound can be easily derived by the use of Euler's formula for planar graphs as follows. Let $E(G)$ be the set of edges of a graph $G$ and $V(G)$ the set of vertices of $G$. Denote the number of elements of a set $S$ by $|S|$.

TheOREm 5: Let $\theta(\mathrm{G})=\mathrm{t}>0$. Then there is a vertex of valency less than or equal to $6 \mathrm{t}-1$ in $\mathrm{G}$. 
Proof: Suppose the smallest valency in $G$ is $m$. Then

$$
|E(G)| \geqslant \frac{1}{2} m|V(G)|
$$

Since $\theta(G)=t$, there exist $t$ planar subgraphs $H_{1}, \ldots, H_{t}$ of $G$ whose union is $G$. By Euler's formula in each planar subgraph,

$$
\left|E\left(H_{i}\right)\right| \leqslant 3\left|V\left(H_{i}\right)\right|-6=3|V(G)|-6,
$$

since we may assume that every $H_{i}$ contains all of the vertices of $G$. Thus,

$$
|E(G)| \leqslant 3 t|V(G)|-6 t
$$

Combining (1) and (2),

$$
{ }_{2}^{\frac{1}{2}} m|V(G)| \leqslant 3 t|V(G)|-6 t .
$$

Rearranging terms,

$$
|V(G)|\left(\frac{1}{2} m-3 t\right) \leqslant-6 t .
$$

Since $|V(G)|$ and $t$ are both positive for $t>0$,

$$
1 / 2 m-3 t<0,
$$

or

$$
m \leqslant 6 t-1
$$

Q.E.D.

Corollary: The connectivity of a graph of thickness $\mathrm{t}$ is less than or equal to $6 \mathrm{t}-1$.

\section{Conjectures}

1. There are no $G_{1}$ and $G_{2}$ with $\theta\left(G_{1}\right)=\theta\left(G_{2}\right)=t$ such that $\theta\left(J\left(G_{1}, G_{2}\right)\right)=t+1$. This conjecture is certainly true for $t=1$. For $t>1$, we have been unable to find a counterexample.

2. We have shown in a previous paper [3] that $K_{4 t-5,4 t-5}$ is $t$-minimal for all $t \geqslant 2$. By taking $J\left(K_{4 t-5,4 t-5}, K_{4 t-5,4 t-5}\right)$ and finding a $t$-minimal subgraph, we obtain a $t$-minimal bipartite graph which is not a complete bipartite graph. It would be interesting for each $t$ to characterize the class of all $t$-minimal bipartite graphs.

The 2-minimal bipartite graphs are easily characterized. Kuratowski [4] proved that every 2minimal graph is homeomorphic to $K_{5}$ or $K_{3,3}$. If $G$ is bipartite, let the vertices of $G$ be partitioned into sets $A$ and $B$ such that all edges of $G$ join a vertex in $A$ with one in $B$. Clearly any path joining a vertex in $A$ with a vertex in $B$ contains an odd number of edges (= even number of vertices), while a path joining a vertex in one of the sets $A$ or $B$ with another vertex in the same set contains an even number of edges (= odd number of vertices). To generate all 2-minimal bipartite graphs, start with one of $K_{5}$ or $K_{3,3}$ and write down a partition of its vertices into two sets. With respect to the given partition, an edge joining two vertices in the same set may be replaced by a path consisting of valence 2 vertices and an even number of edges without destroying 2-minimality. Likewise edges joining vertices in different sets may be replaced by paths consisting of valence 2 vertices and an odd number of edges, or by a single edge. The set of all graphs generated by all such replacements in all such partitionings of both $K_{5}$ and $K_{3,3}$ constitutes the complete set of 2-minimal bipartite graphs. 
3. Let $N(t)$ be the smallest integer such that there exists a $t$-minimal graph having $N(t)$ vertices. We know that $N(2)=5$ (reference [4]) and $N(3)=9$ (reference [6]). Beineke and Harary [1] have shown that $6 t-8 \leqslant N(t) \leqslant 6 t-7$ for all $t \geqslant 4$. Since $\theta\left(K_{22}\right)=4$ (see appendix A), $N(5)=6(5)-7=23$. Beineke has shown [8] that $\theta\left(K_{28}\right)=5$, from which it follows that $N(6)=29$. In accordance with Harary's conjecture [7] that $\theta\left(K_{16}\right)=4$, we conjecture that $N(4)=16$ and $N(t)=6 t-7$ for all $t \geqslant 5$.

4 . For all $n \geqslant N(t)$, there exists a $t$-minimal graph having exactly $n$ vertices.

This conjecture is certainly true for $t=2$. For $t>2$, an immediate consequence of Theorem 5.3 of [5] is that for every $n \geqslant N(t)$, there exists a $t$-minimal graph having at least $n$ vertices. Further evidence about the conjecture is lacking, and it does not appear to be easily proven if true. Unfortunately, because of the difficulty in establishing the thickness and $t$-minimality of most graphs, the conjecture is not likely to be easily disproven either.

\section{Appendix A}

In their paper on the thickness of the complete graph [1], Beineke and Harary were unable to give the thickness of the graphs $K_{n}$ for $n \geqslant 16$ with $n \equiv 4(\bmod 6)$, except for the case of $K_{28}$

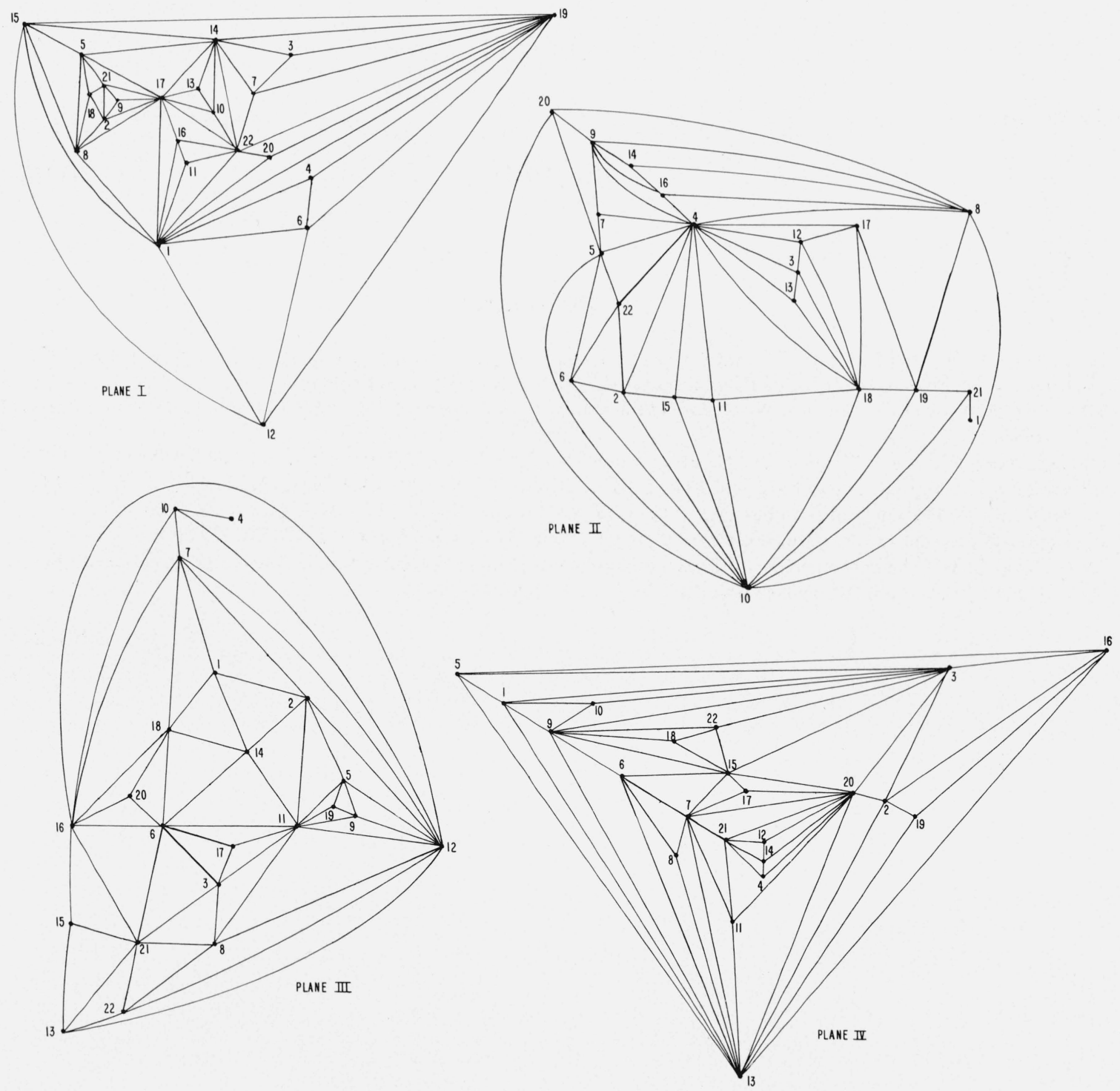

FIGURE 1. $\mathrm{K}_{222}$ in four planes. 
whose thickness they found by trial and error to be 5 . To provide additional data for those who are attempting to determine the thickness of this class of complete graphs, we include the following:

THEOREM: $\theta\left(\mathrm{K}_{222}\right)=4$.

Proof: Figure 1 shows four planar graphs whose union is $K_{22}$. By Euler's formula,

$$
\left|E\left(K_{22}\right)\right| \leqslant 3 t\left|V\left(K_{22}\right)\right|-6 t=60 t .
$$

But

$$
\left|E\left(K_{22}\right)\right|=231
$$

Thus,

$$
60 t \geqslant 231,
$$

or

$$
t \geqslant 4 \text {. }
$$

Therefore,

$$
\theta\left(K_{22}\right)=4
$$

\section{References}

[1] Beineke, L. W., and F. Harary, The thickness of the complete graph, Canadian J. Math. 17, 850-859 (1965).

[2] Dirac, G. A., Short proof of Menger's graph theorem, Mathematika 13, 42-44 (1966).

[3] Hobbs, A. M., and Grossman, J. W., A class of thickness-minimal graphs, J. Res. NBS 72B (Math. Sci.) No. 2, 145-153 (1968).

[4] Kuratowski, C., Sur le Problème des Courbes Gauches en Topologie, Fund. Math. 15, 271-283 (1930).

[5] Tutte, W. T., The thickness of a graph, Nederl. Akad. Wetensch. Proc. Ser. A66 Indag. Math. 25, $567-577$ (1963).

[6] Tutte, W. T., The non-biplanar character of the complete 9-graph, Canad. Math. Bull. 6, 319-330 (1963).

[7] Harary, F., Recent results in topological graph theory, Acta Math. Acad. Sci. Hung. 15, 405-412 (1964).

[8] Beineke, L. W., The decomposition of complete graphs into planar subgraphs, Graph Theory and Theoretical Physics, edited by F. Harary, Academic Press, New York, N.Y., 139-153 (1967).

(Paper 72B3-273) 